ISAF-Streitkräfte zu reduzieren. Das reicht aber noch nicht aus und ist auch nicht mit selbsttragender Sicherheit gleichzusetzen.

Wird Afghanistan jemals imstande sein, seine Bevölkerung zu ernähren und neu geschaffene staatliche Strukturen - mit dem notwendigen Personal, also auch mit Sicherheitskräften - zu bezahlen? Wie im Falle der Polizei besteht die Gefahr, dass Soldaten zu den durch Einnahmen aus dem Drogengeschäft gut ausgestatteten Milizen oder gar zu den Taliban abwandern. Stammeskrieger, die einem archaischen Ehrenkodex verhaftet sind, können nicht kurzerhand zu Staatsbürgern in Uniform verwandelt werden - zumal, wenn ein Staatswesen nur rudimentär vorhanden ist und Uniformen fehlen. Die Wiedereinführung einer allgemeinen Wehrpflicht ${ }^{59}$ wäre eine kostengünstige Alternative und dürfte auch von der Bevölkerung unterstützt werden. Die Afghanen verbinden mit der hoch angesehenen Armee gewissermaßen einen zivilisierenden Einfluss, was auch die Loyalität zum Zentralstaat befördern könnte.

Die internationale Gemeinschaft wird noch einige Jahre und erhebliche Mittel investieren müssen, bevor selbsttragende Sicherheit geschaffen ist. Die Skepsis wird von führenden Offizieren der Bundeswehr geteilt. Zwar halten es einige für möglich,

59 Vgl. Ronald E. Neumann, „Afghans, Report for Duty“, New York Times, 14. Januar 2008, S. A25. in einzelnen Distrikten im Norden die Verantwortung für die Sicherheit afghanischen Kräften zu übertragen. Doch weisen andere darauf hin, dass dies wenig nütze, wenn in den angrenzenden Gebieten weiter Unsicherheit und Gewalt herrschten. Außerdem müssten weiterhin ausländische Kräfte vorgehalten werden, um die Dauerhaftigkeit solch nationaler Lösungen zu gewährleisten. ${ }^{60}$ Schließlich bedarf eine partnerschaftliche Beziehung einer Ausbildung auf Augenhöhe und in angemessenem Tempo, um ANA-Soldaten auch mental aus der Rolle einer Hilfstruppe zu emanzipieren: An Afghan face needs an Afghan pace. Dazu bedarf es strategischer Geduld, politischen Durchhaltevermögens und vorbehaltloser Aufklärung der Bevölkerung in Afghanistan und den ISAF-Entsendestaaten über die Chancen und Risiken des Einsatzes. Die ANA ist auf dem richtigen, wenn auch mühsamen Weg zum Erfolg. Derzeit aber kommt das von NATO-Generalsekretär Anders Fogh Rasmussen apostrophierte Licht am Ende des Tunnels ${ }^{61}$ noch aus den Taschenlampen der eigenen Ausbilder.

60 Siehe Peter Blechschmidt, "Afghanistans erfolglose Armee", Süddeutsche Zeitung, 18. November 2009, S. 1.

61 Anders Fogh Rasmussen, „Afghanistan and NATO: The Way Forward”. Speech by NATO Secretary General at the Atlantic Council of the United States, 28 September 2009, http://www.nato.int/cps/en/natolive/opinions_57722. htm.

\title{
Das Verbot von Chemiewaffen: Fünf Hürden auf dem Weg zu einer chemiewaffenfreien Welt
}

\author{
Thomas Müller-Färber* und Roland Hiemann**
}

\begin{abstract}
This article focuses on the essential challenges to the idea of a world free of chemical weapons. The authors identify five different problem areas carrying the potential to substantially reduce the feasibility of the global prohibition of chemical weapons: the delayed demilitarisation of chemical weapons, related capabilities of non-member states to the Chemical Weapons Convention, the spread and development of non-lethal chemical weapons, the accelerated scientific and technology progress in biochemical sciences and, finally, weak public support for the elimination of chemical weapons. Notwithstanding the substantial progress made in the past, it is argued that the international community needs to tackle these pivotal obstacles in the mid- and long term future in order to achieve a world without chemical weapons.
\end{abstract}

Keywords: Disarmament, chemical weapons, Chemical Weapons Convention (CWC), proliferation Abrüstung, Chemiewaffen, Chemiewaffen-Übereinkommen (CWÜ), Verbreitung

\section{Einführung}

Spätestens seit der Prager Rede von US-Präsident Barack Obama im April 2009, in der er sich für eine Welt ohne Atomwaffen aussprach, hat die Idee von „Global Zero“ verstärkte Aufmerksamkeit erhalten. ${ }^{1}$ Seitdem beherrscht der Gedanke an eine

* Thomas Müller-Färber ist Stipendiat der Friedrich-Ebert-Stiftung und Doktorand an der Eberhard-Karls-Universität Tübingen.

** Roland Hiemann ist Doktorand und Stipendiat der Stiftung Wissenschaft und Politik (SWP) in Berlin.

1 Vgl. Remarks of President Barack Obama (Excerpts), in: Arms Control Today, Mai 2009, http://www.armscontrol.org/node/3644, abgerufen am 19.11.2009.
Welt frei von Kernwaffen viele aktuelle rüstungs- und sicherheitspolitische Debatten. Während sich namhafte Experten in jüngster Vergangenheit mit der Frage auseinandergesetzt haben, wie die Vision einer vollständigen nuklearen Abrüstung gelingen könnte, ${ }^{2}$ ist die „Global Zero“-Idee für Chemiewaffen (CW) bereits in vielerlei Hinsicht Wirklichkeit geworden. CW sind heute mit einem umfassenden, nahezu weltweit gültigen

2 Vgl. Shultz, George P.; Perry, William J.; Kissinger, Henry A; Nunn, Sam: A World Free of Nuclear Weapons, The Wall Street Journal, 04. Januar 2007 Schmidt, Helmut; Weizsäcker, Richard von; Bahr, Egon; Genscher, Hans-Dietrich: Für eine atomwaffenfreie Welt, Frankfurter Allgemeine Zeitung, 09. Januar 2009. 
Tabu belegt, das sowohl völkerrechtlich als auch politisch begründet ist. Beim CW-Tabu handelt es sich um eine soziale Norm, die CW ächtet. ${ }^{3}$

Auch wenn CW seit dem Ersten Weltkrieg in verschiedenen Konflikten des 20. Jahrhunderts eine unheilvolle Rolle gespielt haben, haben diese nicht-konventionelle Waffengattung und die damit verbundenen Verbotsbestrebungen in der Vergangenheit vergleichsweise wenig öffentliche Aufmerksamkeit erfahren. Vor diesem Hintergrund beleuchtet der vorliegende Artikel die aktuellen Entwicklungen des CW-Verbots, wobei vor allem fünf zentrale Hürden ins Blickfeld rücken, die der Staatengemeinschaft auf dem Weg zu einer chemiewaffenfreien Welt im Wege stehen.

Der entscheidende Garant für Verbot, Nichtverbreitung und Zerstörung von CW stellt auf internationaler Ebene das Chemiewaffen-Übereinkommen (CWÜ) dar, das im April 1997 in Kraft getreten ist. ${ }^{4}$ Das CWÜ gilt im Vergleich zu den internationalen Verträgen, die sich der Problematik der Atom- und Biowaffen annehmen, als besonders weit entwickelt und ausgearbeitet. So ist es das einzige internationale Abkommen, das sowohl die Entwicklung, Herstellung und den Erwerb als auch die Weitergabe und den Einsatz einer ganzen Waffenkategorie ausnahmslos und vollständig verbietet, und zur Kontrolle dieses Verbotes ein komplexes und umfassendes Überwachungssystem aufgebaut hat. ${ }^{5}$ Das Verbot von CW stützt sich in erster Linie auf das sogenannte „allgemeine Zweckkriterium“ (Artikel II CWÜ). Demnach ist der Umgang mit chemischen Substanzen verboten, wenn dies in kriegerischer und nicht-friedlicher Absicht geschieht. Ob bspw. ein Programm offensiv und damit völkerrechtlich verboten oder defensiv und damit erlaubt ist, hängt von der Intention ab, mit der bestimmte chemische Stoffe verwendet werden. Indem chemische Kampstoffe vorwiegend über den Zweck ihrer Anwendung identifiziert werden, wirkt die Verbotsvorschrift des CWÜ prinzipiell allumfassend.

Inspektorenteams kontrollieren sowohl die Zerstörung ehemaliger CW-Bestände als auch Einrichtungen, die zur Entwicklung und Herstellung von CW geeignet sind. Die Überwachung des Chemiewaffen-Verbots wird von der OVCW (Organisation für das Verbot von Chemiewaffen) mit Sitz in Den Haag weltweit organisiert und gesteuert. ${ }^{6}$ Das CWÜ hat in seinem nunmehr zwölfjährigen Bestehen eine erhebliche geographische Ausdehnung erfahren und besitzt heute annähernd globale Geltung. Mittlerweile sind 188 Staaten dem CWÜ beigetreten (Stand November 2009). ${ }^{7}$

Trotz dieser zahlreichen positiven Entwicklungen, die im Wesentlichen auf den Errungenschaften des CWÜ basieren, ist das

3 Vgl. Price, Richard: A Genealogy of the Chemical Weapons Taboo, in: International Organization 49. Jg. (1995), Nr. 1, S. 73-103.

4 Vgl. Übereinkommen über das Verbot der Entwicklung, Herstellung, Lagerung und des Einsatzes chemischer Waffen und über die Vernichtung solcher Waffen, http://www.auswaertiges-amt.de/diplo/de/Aussenpolitik/Themen/Abruestung/Downloads/CWUE.pdf, abgerufen am 19.11.2009.

5 Vgl. Thränert, Oliver; Tucker, Jonathan B.: Freeing the World of Chemical Weapons. The Chemical Weapons Convention at the Ten-year Mark, (SWP Research Paper, Nr. 8), Juli 2007, S. 10.

6 Vgl. Kelle, Alexander; Nixdorff, Kathryn; Dando, Malcolm: Controlling Biochemical Weapons. Adapting Multilateral Arms Control for the 21st Century, Hampshire/New York 2006.

7 Vgl. OVCW, http://www.opcw.org/about-opcw/member-states/, abgerufen am 30.11.2009. Als 188. Staat sind die Bahamas mit Wirkung vom 21. Mai 2009 dem CWÜ beigetreten. weltweite Verbot von CW dennoch mit einigen Problemen behaftet. Viele Schwachstellen des CWÜ können potenziell durch einen administrativen, technischen und finanziellen Mehraufwand weitgehend behoben werden. $\mathrm{Zu}$ diesen kurz- und mittelfristig lösbaren Herausforderungen zählt beispielsweise die Implementierung der vertraglichen Bestimmungen und Verpflichtungen des CWÜ, die in einigen Mitgliedstaaten noch lückenhaft ist, oder der noch ausstehende Beitritt einiger kleiner Staaten, die mit großer Wahrscheinlichkeit keine CW besitzen und die bisher dem CWÜ aufgrund mangelnder technischer, administrativer und finanzieller Kompetenzen ferngeblieben sind. Zu diesen Staaten gehören bspw. Somalia oder Angola.

Neben solchen grundsätzlich behebbaren Schwächen im CWÜ ist die CW-Prohibition jedoch auch mit Herausforderungen konfrontiert, die ein deutlich größeres sicherheitspolitisches Gewicht aufweisen und deren Bewältigung die Staaten, die sich der Idee einer chemiewaffenfreien Welt verpflichtet haben, vor erhebliche Schwierigkeiten stellt. Es handelt sich dabei im Wesentlichen um fünf überaus unterschiedlich gelagerte Problembereiche, denen wiederum spezifische Ursachen zugrundeliegen, die jedoch alle das Potenzial haben, die Leistungsfähigkeit des internationalen CW-Verbots dauerhaft zu untergraben: 1. die nicht fristgerechte Zerstörung bestehender CW-Bestände, 2. die mit CW gerüsteten Nicht-Mitgliedstaaten, 3. die Problematik „nicht-tödlicher" CW, 4. der beschleunigte wissenschaftlich-technologische Fortschritt und 5. die mangelnde öffentliche Unterstützung für die Vision eines umfassenden weltweiten Verbots von CW. Bedauerlicherweise verhalf auch die zweite Überprüfungskonferenz des CWÜ, die im April 2008 in Den Haag stattfand, zu keinem wesentlichen Fortschritt bei der Überwindung dieser Hürden, die auf dem Weg zu einer vollkommen $\mathrm{CW}$-freien Welt genommen werden müssen.

\section{Nicht-fristgerechte Zerstörung der Chemie- waffen-Arsenale}

Die nicht-fristgerechte Zerstörung der deklarierten CW-Bestände stellt ein zentrales Problem der CW-Prohibition dar. Alle Mitgliedstaaten des CWÜ haben sich mit der Unterzeichnung dieses Abkommens verpflichtet, sämtliche $\mathrm{CW}$, die sich in ihrem Besitz befinden, zu deklarieren und innerhalb einer Frist von zehn Jahren - also bis April 2007 - vollständig zu vernichten. Von den sieben Mitgliedstaaten, die den Besitz von CW offiziell eingeräumt haben, konnte bisher nur Albanien dieser Verpflichtung (mit einer moderaten Fristüberschreitung) nachkommen. ${ }^{8}$ Im Sommer 2008 gelang es schließlich auch Südkorea, seine CW-Arsenale vollkommen zu vernichten. ${ }^{9}$ Die anderen Mitgliedstaaten, die den Besitz von CW eingeräumt haben - Indien, Libyen, Russland, Südkorea und die USA - erhielten einen Aufschub von bis zu fünf Jahren. Darüber hinaus bestehen Unklarheiten, ob der Irak, der im Februar 2009 Mit-

8 Vgl. OVCW: Albania - First Country to Destroy All of its Chemical Weapons, in: OPCW Press Release Nr. 73, Den Haag, 12. Juli 2007, http://www.opcw. org/news/news/article/albania-the-first-country-to-destroy-all-its-chemicalweapons/, abgerufen am 19.11.2009.

9 Vgl. South Korea Completes Destruction of Its Chemical Weapons Stockpile, in: Environmental News Service, 17. Oktober 2008, http://www.ens-newswire. com/ens/oct2008/2008-10-17-03.asp, abgerufen am 19.11.2009. 
glied des CWÜ geworden ist, seine deklarierten CW-Bestände fristgerecht zerstören kann. Bisher ist noch nicht einwandfrei geklärt, wie umfassend die irakischen CW-Bestände, die in zwei Bunkern lagern, sind. ${ }^{10}$

Besonderes Kopfzerbrechen bereitet die schleppende Zerstörung der CW-Arsenale in den USA (ca. 30.000 Tonnen) und in Russland (ca. 40.000 Tonnen). Diese beiden CWÜ-Mitgliedstaaten verfügen insgesamt über ca. 98 Prozent der weltweit offiziell deklarierten CW-Bestände. ${ }^{11}$ Trotz der verlängerten Frist bis zum April 2012 werden die USA und Russland die vertraglich fixierte Verpflichtung zur vollkommenen chemischen Demilitarisierung aller Wahrscheinlichkeit nach nicht einhalten können. Verantwortlich für die zögerliche Zerstörung der CW-Arsenale sind in erster Linie technische und finanzielle, aber auch politische Schwierigkeiten, die unmittelbar bei der Vernichtung dieser Waffenbestände entstehen. ${ }^{12}$

Zwar erklärte das US-amerikanische Verteidigungsministerium im November 2008 , dass ca. 58 Prozent der ursprünglich deklarierten chemischen Kampfstoffe vernichtet worden seien, doch die vollkommene Eliminierung der verbleibenden CWBestände wird voraussichtlich erst in den Jahren 2015 oder 2017 beendet sein. ${ }^{13}$ Es sind aber auch Szenarien denkbar, in denen die Vernichtung der chemischen Kampfstoffe in den USA erst im Jahre 2020 oder 2023 abgeschlossen sein wird. ${ }^{14}$ Trotz internationaler Hilfestellung - sowohl technischer als auch finanzieller Art - ist stark davon auszugehen, dass Russland ebenfalls nicht in der Lage sein wird, die restlichen zwei Drittel seines CW-Arsenals fristgerecht zu vernichten. ${ }^{15}$

Für die generelle Leistungsfähigkeit des Verbots von CW besteht das Problem der schleppenden CW-Vernichtung weniger in der Gefahr, dass diese Kampfstoffe tatsächlich zum Einsatz kommen. In Moskau ebenso wie in Washington scheint der politische Wille zur vollkommenen Zerstörung dieser Waffen grundsätzlich vorhanden zu sein. Zudem werden die deklarierten chemischen Kampfstoffe und deren Vernichtung von den Inspektoren der OVCW überwacht. ${ }^{16}$ Die militärische Reaktivierung dieser Bestände durch die Mitgliedstaaten ist somit weitgehend ausgeschlossen. Vielmehr stellt die verzögerte Vernichtung aufgrund des daraus resultierenden Regel- und Normenverstoßes ein direktes Problem für die Stabilität und Glaubwürdigkeit der weltweiten Tabuisierung derartiger Waffen dar. Kommt es im Jahre 2012 zu Vertragsverletzungen, werden das

10 Vgl. OVCW: Opening Statement by the Director-General To The Executive Council at its fifty-sixth Session, 21.-24. April 2009, http://www.opcw.org/ fileadmin/OPCW/EC/56/en/ec56dg10_en.pdf, abgerufen am 19.11.2009.

11 Vgl. OVCW, http://www.opcw.org/our-work/demilitarisation/, abgerufen am 19.11.2009.

12 Zum einen sind für die Verzögerungen in den USA die hohen Kosten verantwortlich, die bei der chemischen Demilitarisierung anfallen. Zum anderen wird die Beseitigung chemischer Kampfstoffe auch durch den Widerstand von Anwohnern und Umweltgruppen behindert. Vgl. Tucker, Jonathan B.: War of Nerves. Chemical Warfare from WW I to al-Qaeda. New York/Toronto 2006. S. 383 . Chemical Warfare from WW I to al-Qaeda. New York/Toronto 2006. S. 383

13 Vgl. Meier, Oliver: U.S., Russia Step Up Chemical Weapons Destruction, in: Arms Control Today, Dezember 2008.

14 Vgl. Krieg, Kenneth J. (Under Secretary of Defense): Memorandum for Program Manager, Assembled Chemical Weapons Alternatives Deputy Under Secretary of Defense (Installations and Environment) Director, Defense Contract Management Agency (10. Januar 2007).

15 Im März 2009 wurde eine neue Vernichtungsanlage in Shchuch'ye in Betrieb genommen. Vgl. Arnaudo, Daniel: CW Destruction Site Begins Operation, in: Arms Control Today, April 2009.

16 Vgl. Thränert; Tucker: a.a.O., S. 18.
Verbotsbestreben und somit auch das CWÜ - unabhängig davon, welche technischen und völkerrechtlichen Lösungen vorhanden sind - mit großer Wahrscheinlichkeit vor ein schwer lösbares politisches Dilemma gestellt. Mitgliedstaaten, die nach dem April 2012 Vertragsverletzungen begehen, werden mit Hinweis auf den Verstoß seitens der USA und Russlands bei der Vernichtung ihrer CW auf eine wohlwollende Bewertung ihrer vertraglichen Nichteinhaltung pochen. Dies könnte die Wirksamkeit des CW-Verbots sichtbar schwächen. Gesetzt den Fall, dass die Mehrheit der OVCW-Mitglieder den Forderungen einzelner Staaten nach einer wohlgesinnten, konzilianten Auslegung ihrer etwaigen Normen- und Regelverstöße nicht nachkommt, könnte dies den Vorwurf der ungerechten ZweiKlassen-Mitgliedschaft provozieren. Bereits in der Vergangenheit haben einige Staaten - wie bspw. der Iran - bewiesen, dass sie durch den lautstarken Verweis auf die mangelnde Fairness internationaler Vertragswerke politikwirksame Turbulenzen in der Rüstungskontrolle erzeugen können. ${ }^{17}$

Vor dem Hintergrund dieser vertraglichen Erschütterungen und den daraus resultierenden Konsequenzen für die Idee einer CW-freien Welt tragen die betreffenden CW-Staaten eine besondere Verantwortung, die fristgerechte Zerstörung ihrer deklarierten chemischen Kampfstoffe - unter Einhaltung allgemeiner Umwelt- und Gesundheitsstandards - zu gewährleisten.

\section{Chemiewaffen-gerüstete Nicht-Mitglied- staaten}

Einer Reihe von Regionalmächten, die bisher dem CWÜ ferngeblieben ist, wurde mehrfach vorgeworfen, CW zu besitzen. $\mathrm{Zu}$ diesen Nicht-Mitgliedstaaten zählen Ägypten, Israel, Nordkorea und Syrien. Mit CW gerüstete Nicht-Mitgliedstaaten stellen eine bedeutende sicherheitspolitische Hürde auf dem Weg zu einer CW-freien Welt dar.

Ägypten setzte während des jemenitischen Bürgerkrieges (19631967) chemische Kampfstoffe ein. Die damals verwendeten CW stammten aller Wahrscheinlichkeit nach aus Arsenalen der britischen Kolonialtruppen. Konservativen Schätzungen zufolge kamen durch diesen CW-Einsatz 1.500 Menschen ums Leben. In den 1960er Jahren hat Ägypten begonnen, eigene CW-Kapazitäten zu entwickeln. Im Vorfeld des Jom-Kippur-Krieges 1973 wurden chemische Sprengkörper vermutlich nicht nur stationiert, sondern auch an Syrien transferiert. Aus Furcht vor Israels nuklearer Vergeltung setzten damals weder Ägypten noch Syrien diese Kampfstoffe ein. ${ }^{18}$ Zwar bleibt der genaue Umfang des ägyptischen CW-Programms unklar, doch liegt die Vermutung nahe, dass Ägypten - wie auch Syrien - seine chemische Schlagkraft als „Ersatz“ für die nicht zu erreichende nukleare Waffenoption betrachtete sowie als geeignetes Instrument der nicht-konventionellen Abschreckung, um Israels Kernwaffenpotenzial strategisch zu neutralisieren. Doch auch politische

17 Ob der Vorwurf der „Doppelstandards“ durch die ernst gemeinte Besorgnis um die Leistungsfähigkeit des entsprechenden internationalen Abkommens (insbesondere des Atomwaffensperrvertrages) oder durch strategische Nationalinteressen verursacht wird, sei hier dahingestellt.

18 Vgl. Burck, Gordon M.; Flowerree, Charles C.: International Handbook on Chemical Weapons Proliferation. New York 1991, S. 105. 
Motive scheinen stets eine gewichtige Rolle gespielt zu haben, zumal Ägypten und Syrien ihre CWÜ-Mitgliedschaft nach wie vor vom Beitritt Israels zum Atomwaffensperrvertrag abhängig machen. ${ }^{19}$

Israel hat vermutlich unmittelbar nach der Erlangung seiner Unabhängigkeit Ende der 1940er Jahre ein Programm zur Entwicklung und Herstellung von chemischen Kampfstoffen aufgenommen. ${ }^{20} \mathrm{Ob}$ und in welchem Ausmaß das Land noch heute über offensive CW verfügt, ist strittig, zumal auch sie (ähnlich wie die israelischen Atomwaffen) von einem Mantel der Verschwiegenheit umgeben sind. Es bietet sich die Interpretation an, dass Israel sein CW-Programm anfangs lediglich als eine Art strategische „Zwischenlösung“ auf dem Weg zum ultimativen Abschreckungspotenzial - der Atombombe - betrachtet hat. Wahrscheinlich misst Israel seiner offensiven CWKapazität seit der Verfügung über nukleare Fähigkeiten im Jahre 1967 nur noch geringe Bedeutung bei. ${ }^{21}$

Nach Einschätzung westlicher Geheimdienste zählt das CWArsenal Nordkoreas zu den weltweit größten. Wahrscheinlich baute das Land bereits in den 1950er Jahren mit Hilfe der Sowjetunion ein offensives CW-Programm auf. Heute sollen dem nordkoreanischen Regime zwischen 2.500 und 5.000 Tonnen chemische Kampfstoffe zur Verfügung stehen. ${ }^{22}$

Das syrische CW-Programm muss - ähnlich wie im Falle Ägyptens - im Kontext der regionalen Rüstungspolitik gesehen werden. Damaskus hat mehrmals betont, dass syrische CW in erster Linie dazu dienen, der atomaren Schlagkraft Israels entgegenzutreten. ${ }^{23}$ Während Syrien anfänglich seine CW von Ägypten erhielt, begann das Land wahrscheinlich ab den 1970er Jahren eigene CW-Kapazitäten aufzubauen und ist heute vermutlich im Besitz von 500 bis 1.000 Tonnen chemischer Substanzen, die zu Waffenzwecken verwendet werden könnten. ${ }^{24}$ Damit besitzt Syrien heute laut US-Angaben das umfassendste und modernste Chemiewaffen-Arsenal im Nahen Osten. ${ }^{25}$

Unser Wissen über die tatsächlichen rüstungspolitischen Abläufe im Zusammenhang mit chemischen Kampfstoffen bleibt nach wie vor vage und lückenhaft. $\mathrm{Zu}$ vermuten ist jedoch: Gelingt es, die verbleibenden Nicht-Mitgliedstaaten, die aller Wahrscheinlichkeit nach über CW verfügen, in die Bemü-

19 Vgl. Shoham, Dany: Chemical and Biological Weapons in Egypt, in: The Nonproliferation Review, 5. Jg. (1998) Nr. 3, S. 52-53.

$20 \mathrm{Vgl}$. Cohen, Avner: Israel and Chemical/Biological Weapons: History, Deterrence, and Arms Control, in: The Nonproliferation Review, 8. Jg. (2001) Nr. 3, S. 27-53, S. 29-34

21 Vgl. Cirincione, Joseph; Wolfsthal, Jon B.; Rajkumar, Miriam: Deadly Arsenals. Nuclear, Biological and Chemical Threats, Washington (2. Aufl.) 2005, S. 259-275.

22 Vgl. The International Institute for Strategic Studies: North Korea's Chemical and Biological Weapons (CBW) Programmes, S. 49, http://www.iiss.org/EasysiteWeb/getresource. axd?AssetID=1869\&type=full\&servicetype=Attachm ent, abgerufen am 19.11.2009; vgl. außerdem den Bericht der International Crisis Group: North Korea's Chemical and Biological Weapons Programs (Asia Report Nr. 167 - 18. Juni 2009), S. 7, http://www.crisisgroup.org/library/documents/asia/north_korea/167_north_koreas_chemical_and_biological_weapons_programs.pdf, abgerufen am 19.11.2009.

23 Vgl. Diab, Zuhair: Syria's Chemical and Biological Weapons. Assessing Capabilities and Motivations, in: The Nonproliferation Review, 5. Jg. (1997), Nr. 1 , S. 104-111, S. 105

24 Vgl. Cordesman, Anthony H.: Weapons of Mass Destruction in the Middle East: Regional Trends, National Forces, Warfighting Capabilities, Delivery Options, and Weapon Effects, Center for Strategic and International Studies (CSIS), Washington, D.C. 2003, S. 55f.

25 Vgl. Thränert, Oliver: Zehn Jahre Chemiewaffen-Verbot. Erfahrungen und Perspektiven, in: Europäische Sicherheit, April 2007. hungen um ein umfassendes CW-Verbot einzubinden, würde dies die Leistungsfähigkeit der CW-Prohibition erheblich erhöhen. Zudem gelten starke multilaterale Rüstungskontrollverträge mit einer umfassenden Mitgliedschaft als ein wirksames Instrument der Terrorismusbekämpfung - ein Aspekt, der im Zusammenhang mit chemischen Waffen besonders brisant erscheint. $^{26}$

\section{4. "Nicht-tödliche“ Chemiewaffen}

„Nicht-tödliche“ CW, worunter eine Reihe von Kampfstoffen wie etwa Tränengas fallen, stellen ein weiteres Problem der Verbotsbestrebungen dar. Das CWÜ lässt zum Zweck der Aufrechterhaltung der öffentlichen Ordnung - bspw. zur Bewältigung innerstaatlicher Unruhen - die Verwendung von chemischen Substanzen zu. ${ }^{27}$ Innerhalb des CWÜ handelt es sich dabei um die bedeutendste Ausnahme vom generellen CW-Verbot. Es ist allerdings fraglich, ob sich die Aufrechterhaltung der öffentlichen Ordnung nur auf den innerstaatlichen Bereich bezieht, oder ob die Verwendung von „nicht-tödlichen“ CW auch in internationalen Konflikt- und Krisenszenarien - wie bspw. bei multilateralen Friedenseinsätzen - vom CWÜ abgedeckt wird. Das internationale CW-Verbot ist diesbezüglich nicht eindeutig und erlaubt hier Interpretationsspielraum, der in den letzten Jahren einige Debatten ausgelöst hat. ${ }^{28}$

Auf der einen Seite kann eingeräumt werden, dass die Verwendung von „nicht-tödlichen“ CW in Kriegs- und Krisensituation einen verhältnismäßigen Vorteil verspricht. Schließlich könnte dadurch das Eskalationsrisiko gesenkt und die Zahl der Opfer reduziert werden, die bei der Anwendung von weitaus destruktiveren konventionellen Waffensystemen entstehen würde. Von dieser Perspektive aus betrachtet ist die Entscheidung nachvollziehbar, die Bundeswehr für ihre Auslandseinsätze mit Tränengas auszurüsten. Dass „nicht-tödliche“ CW nun der Bundeswehr auch im Rahmen von Auslandseinsätzen zur Verfügung stehen, ist dabei den Erfahrungen geschuldet, die im März 2004 bei Unruhen der albanischen Bevölkerungsmehrheit im Kosovo gemacht wurden. ${ }^{29}$

Auf der anderen Seite führen Kritiker an, dass die Verwendung von „nicht-tödlichen“ CW ein beachtliches Schlupfloch innerhalb des Vertrages darstellt. Es wird in diesem Zusammenhang auf die Gefahr hingewiesen, dass unter dem Vorwand, lediglich die erlaubte Kategorie der „nicht-tödlichen“ Kampfstoffe herzustellen, Waffen eines neuen Typs entwickelt werden könnten, die das Kriegsgeschehen verändern und zudem neue Felder der internationalen Rüstungsdynamik eröffnen könnten. Diese Befürchtung wird durch die Tatsache unterstrichen, dass einige Staaten - allen voran die USA und Russland - in den letzten

26 Vgl. Tucker, Jonathan B.: Bridging the Gaps: Achieving the Potential of the Nonproliferation Treaties to Combat Nuclear, Biological, and Chemical Terrorism, in: Die Friedenswarte 83. Jg. (2008) Nr. 2-3, S. 81-103.

27 Vgl. CWÜ Artikel II, Absatz 9.

28 Vgl. Ballard, Kyle M.: Convention in Peril? Riot Control Agents and the Chemical Weapons Ban, in: Arms Control Today, September 2007; außerdem Dando, Malcolm: Scientific and Technological Change and the Future of the CWC: The problem of Non-lethal Weapons, in: Disarmament Forum, Nr. 4, 2002, S. 33-44, S. 33-36.

29 Vgl. Knauer, Sebastian: Chemiewaffen für die Bundeswehr, in: Der Spiegel, 31.Juli 2004 
Jahren ein verstärktes Interesse an diesem Waffentyp gezeigt haben. ${ }^{30}$

Gegen den Einsatz von „nicht-tödlichen“ CW wird zudem immer wieder eingewandt, dass damit das allgemeine internationale CW-Tabu untergraben würde, auf dem letztendlich das Vertragswerk des CWÜ basiert. Der Einsatz von „nicht-tödlichen" CW könnte die Grenzen dieser Norm aufweichen und somit deren Bedeutung reduzieren.

Ein weiteres Problem besteht darin, dass die Grenze zwischen tödlichen und „nicht-tödlichen“ chemischen Kampfstoffen in der Realität oft nur schwer gezogen werden kann. Ob bestimmte chemische Substanzen letale Wirkung haben, hängt von verschiedenen Faktoren ab wie dem Alter, dem Gesundheitszustand und dem Grad der Exponiertheit der Opfer, aber auch von Umweltbedingungen, die zum Zeitpunkt der Ausbringung der entsprechenden Substanzen herrschen. ${ }^{31}$ In diesem Zusammenhang wird oftmals auf die Geiselbefreiung in einem Moskauer Theater durch russische Sicherheitskräfte im Jahre 2002 verwiesen. Beim Einsatz von Fentanyl, einem synthetischen Opiat, das damals als „nicht-tödlicher“ Kampfstoff verwendet wurde, kamen 117 Geiseln sowie 50 tschetschenische Geiselnehmer ums Leben. ${ }^{32}$ Allerdings ist bis heute nicht eindeutig geklärt, wie viele der Kidnapper damals tatsächlich durch die Wirkung des Gases starben und wie viele von russischen Sicherheitskräften erschossen wurden. ${ }^{33}$

Schließlich führt eine historische Betrachtungsweise zu der Erkenntnis, dass „nicht-tödliche“ CW in den meisten kriegerischen Auseinandersetzungen dazu verwendet wurden, um konventionellen Waffen ein noch höheres Tötungspotenzial zu verleihen. ${ }^{34}$ In allen Konflikten seit dem Ersten Weltkrieg, in dem CW zum Einsatz gekommen sind - bspw. im irakisch-iranischen Krieg (1980-1988) oder im jemenitischen Bürgerkrieg (1963-1967) - hat die Anwendung von Tränengas den Auftakt zum Einsatz von tödlichen CW gegeben. Historisch gesehen wird also der Einstieg zur CW-Kriegführung in der Regel über die Anwendung von „nicht-tödlichen“ Kampfmitteln beschritten. ${ }^{35}$

Um also die Leistungsfähigkeit des generellen CW-Verbots zu erhöhen, muss der völkerrechtliche Graubereich, der hinsichtlich der Anwendung von „nicht-tödlichen“ CW besteht, dringend bereinigt werden.

30 Vgl. Seidler-Diekmann, Tabea: Alte Probleme in neuem Gewand? Herausforderungen an das umfassende Chemiewaffenverbot, in: HSFK-Report, 2008, Nr. 2, S. 25 / Sutherland, Ronald G.: Chemical and Biochemical Non-lethal Weapons. Political and Technical Aspects, SIPRI Policy Paper, Nr. 23, 2008, S. $10-11$.

31 Vgl. Klotz, Lynn; Furmanski, Martin; Wheelis, Mark: Beware the Siren's Song. Why "Non-Lethal" Incapacitating Chemical Agents are Lethal, 2003, www. fas.org/bwc/papers/sirens_song.pdf, abgerufen am 19.11.2009.

32 Vgl. Pearson, Alan M.: Incapacitating Biochemical Weapons. Science, Technology, and Policy for the 21st Century, in: The Nonproliferation Review, 13. Jg. (2006) Nr. 2, S. 152.

33 Vgl. BBC News: Gas killed Moscow hostages, 27. Oktober 2002, http://news. bbc.co.uk/2/hi/europe/2365383.stm, abgerufen am 19.11.2009.

34 Vgl. Herby, Peter: Protecting and Reinforcing Humanitarian Norms. The Way Forward. in: Peterson, Alan M.; Chevrier, Marie Isabelle; u.a. (Hrsg.): Incapacitating Biochemical Weapons. Lanham 2007, S. 285-291, S. 287 / SeidlerDiekmann: a.a.O., S. 26.

35 Vgl. Meselson, Matthew; Robinson, Julian: „Non-lethal“ Weapons, the CWC and the BWC, in: The CBW Conventions Bulletin, Nr. 61, September 2003, S. 2 .

\section{Beschleunigter wissenschaftlich-technolo- gischer Fortschritt}

Eine generelle Problematik im Bereich der Massenvernichtungswaffen, mit der auch die Verbotsbestrebungen von CW konfrontiert sind, stellt der rasante wissenschaftlich-technologische Fortschritt dar, der - beabsichtigt oder nicht - stetig neue potenziell waffentaugliche Substanzen, Technologien und entsprechendes Know-How erzeugt. Das heißt: Viele Errungenschaften der biochemischen Wissenschaft haben eine „doppelte Verwendungsmöglichkeit“ (dual use). Es besteht demnach grundsätzlich die Möglichkeit, sie sowohl für friedliche und zivile als auch für kriegerische Zwecke einzusetzen. ${ }^{36}$

Ähnlich wie die entsprechenden internationalen Abkommen im Bereich der Atom- und Biowaffen, fällt auch dem CWÜ die diffizile Aufgabe zu, diese wissenschaftlich-technologische Entwicklung zu kontrollieren und eventuell zu beschränken, ohne dabei jedoch die positiven Effekte des wissenschaftlichen und industriellen Chemiesektors „abzuwürgen“. Dabei steht das CW-Verbot jedoch vor erheblichen Schwierigkeiten, insbesondere, weil sich der wissenschaftlich-technologische Fortschritt im Chemiesektor in den letzten Jahren zunehmend beschleunigt und ausdifferenziert hat. Besonders im Bereich der Bio- und Nanotechnologie sind Ergebnisse erzielt worden, die ein hohes CW- Potenzial zu besitzen scheinen. ${ }^{37}$ Zudem ist der Widerstand von Seiten der Wissenschaft und der chemischen Industrie gegen eventuelle regulierende Eingriffe groß. Darüber hinaus hat sich die Struktur der Chemieindustrie in den letzten Jahren zunehmend verändert. Mehr und mehr gewinnen kleinere, flexiblere, den Entwicklungen des Marktes besser angepasste und die Lagerkosten reduzierende Produktionsweisen an Bedeutung. Diese sind jedoch immer schwieriger zu kontrollieren. ${ }^{38}$ Ferner ist die chemische Forschung und Industrie nicht mehr nur auf die westlichen Industrieländer begrenzt. Schwellenländer und Staaten im Umbruch haben vermehrt in den Auf- und Ausbau dieses Sektors investiert. Dementsprechend hat der weltweite Handel mit chemischen Substanzen erheblich zugenommen. ${ }^{39}$

Dies alles erschwert der OVCW die weltweite Kontrolle und Überwachung. Der wissenschaftlich-technologische Fortschritt hat somit das Potenzial, die Bedeutung des CW-Tabus und somit die des CWÜ zu untergraben, da das potenzielle Risiko von Regelverstößen kontinuierlich wächst.

\section{Mangelnde öffentliche Unterstützung}

Die geringe öffentliche Aufmerksamkeit und Unterstützung stellen die fünfte Hürde auf dem Weg zu einer vollständig CW-freien Welt dar. Vor allem im Vergleich zum Themenfeld Atomwaffen sticht das geringe Interesse der Öffentlichkeit am CW-Verbot besonders ins Auge. Beim globalen Verbot von CW sind bis heute die Debatteneuphorie und Initiativfreudigkeit

\footnotetext{
36 Vgl. Robinson, Perry J.P.: Difficulties Facing the Chemical Weapons Convention, in: International Affairs, 84. Jg. (2008) Nr. 2, S. $227 \mathrm{f}$.

37 Vgl. Trapp, Ralf: Advances in Science and Technology and the Chemical Weapons Convention, in: Arms Control Today, März 2008.

38 Vgl. Seidler-Diekmann, a.a.O., S. 10-11.

39 Vgl. Trapp, a.a.O.
} 
zu vermissen, wie sie sich aktuell bei der Debatte um eine kernwaffenfreie Welt abzeichnen. Es existieren nur wenige zivilgesellschaftliche Akteure, die sich in diesem Zusammenhang engagieren. ${ }^{40} \mathrm{Nach}$ wie vor wird das internationale CW-Verbot in erster Linie von den Mitarbeitern der OVCW und den Beamten der Mitgliedstaaten getragen und umgesetzt.

Auch die Medien in den Mitgliedstaaten - ausgewiesene Fachzeitschriften ausgenommen - interessieren sich gewöhnlich kaum für dieses sicherheitspolitische Thema. ${ }^{41}$ Zudem sind vereinzelt Zweifel angebracht, ob sich Forschung und Industrie des biochemischen Sektors - Ausnahmen mitberücksichtigt - über die Problematik der CW und ihrer eigenen möglichen Schlüsselrolle bei der Entwicklung und Herstellung von potenziell CW-tauglichen Substanzen und Technologien bewusst sind. ${ }^{42}$

Die positiven Erfahrungen, die vor allem in der konventionellen Rüstungskontrolle - insbesondere im Bereich der Antipersonenminen und Streumunition - gemacht wurden, zeigen, wie wichtig und gewinnbringend eine umfassende Unterstützung von Seiten einer interessierten Öffentlichkeit sein kann. ${ }^{43}$ Nichtregierungsorganisationen kontrollieren und überwachen die Aktivitäten der Mitgliedstaaten, tragen zur Schaffung eines allgemeinen Problembewusstseins bei und bauen auf der innenpolitischen Ebene der Mitgliedstaaten Druck auf, der die multilateralen Verhandlungen auf internationaler Ebene entsprechend beeinflusst. Die Leistungsfähigkeit von sicherheitspolitischen Abkommen wird dadurch deutlich erhöht. ${ }^{44}$

\section{Fazit}

Auch wenn festgehalten werden kann, dass das Verbot von CW seit dem nunmehr zwölfjährigen Bestehen des CWÜ eine Reihe von beachtlichen Fortschritten erzielt hat, so ist es doch verfrüht, diese bedeutenden sicherheitspolitischen Verbotsanstrengungen als reine Erfolgsgeschichte zu bezeichnen. Denn die hier beschriebenen fünf Problembereiche haben durchaus das Potenzial, die Leistungsfähigkeit der CW-Tabuisierung und damit die Wirksamkeit des CWÜ mittel- und langfristig zu schmälern. Im Umkehrschluss bedeutet dies: Der Weg zu einer Welt, die vollkommen von CW befreit ist, kann nur dann erfolgreich beschritten werden, wenn es der internationalen Gemeinschaft gelingt, diese Schwachstellen zu beseitigen.

Leider wurden diese zentralen Probleme auf der Zweiten Überprüfungskonferenz des CWÜ im Frühjahr 2008 nicht bzW. angesichts ihrer sicherheitspolitischen Brisanz nicht ausreichend

40 Bspw. die "International Union of Pure and Applied Chemistry" oder "Green Cross".

41 Vgl. Dando, Malcolm: Missed Opportunities at the Chemical Weapons Treaty Meeting, in: Bulletin of Atomic Scientists, Nr. 12, Mai 2008.

42 Vgl. Dando, Malcolm R.; Rappert, Brian: Codes of Conduct for the Life Sciences. Some Insights form UK Academia, in: Department of Peace Studies University of Bradford, Strengthening the Biological Weapons Convention, Briefing Paper (Second Series) Nr. 16, Mai 2005.

43 Vgl. Bleicher, Maurice: The Ottawa Process. Nine-day Wonder or Model for Disarmament Negotiations?, in: Disarmament Forum, Nr. 2, 2000, S. 69-86/ Justen, Detlev: Der Oslo-Prozess zum Verbot von Streumunition. Die Stigmatisierung von „Cluster Bombs" hat begonnen, in: SWP-Studie, Nr. 30, Berlin, Oktober 2008 .

44 Vgl. Haas, Peter M.: Introduction. Epistemic Communities and International Policy Coordination, in: International Organization, 46. Jg. (1992), Nr. 1, S. 1-35 / Keck, Magaret; Sikkink, Kathryn: Activists Beyond Borders. Advocacy Networks in International Politics, Ithaca, 1998. bearbeitet und diskutiert. ${ }^{45}$ Damit wurde eine entscheidende Chance für eine forcierte Lösung der Schwachstellen des CWVerbots vertan. Es bleibt zu hoffen, dass die dritte Überprüfungskonferenz, die für das Jahr 2013 angesetzt ist, sinnvoll genutzt wird, um diese virulenten Probleme des CWÜ umfassend und nachhaltig zu lösen.

\section{Literatur}

Arnaudo, Daniel: CW Destruction Site Begins Operation, in: Arms Control Today, April 2009.

Ballard, Kyle M.: Convention in Peril? Riot Control Agents and the Chemical Weapons Ban, in: Arms Control Today, September 2007.

BBC News: Gas killed Moscow hostages, 27. Oktober 2002, http://news.bbc.co.uk/2/hi/europe/2365383.stm, abgerufen am 19.11.2009.

Bleicher, Maurice: The Ottawa Process. Nine-day Wonder or Model for Disarmament Negotiations?, in: Disarmament Forum, Nr. 2, 2000, S. 69-86.

Burck, Gordon M.; Flowerree, Charles C.: International Handbook on Chemical Weapons Proliferation. New York 1991.

Cirincione, Joseph; Wolfsthal, Jon B.; Rajkumar, Miriam: Deadly Arsenals. Nuclear, Biological and Chemical Threats, Washington (2. Aufl.) 2005.

Cohen, Avner: Israel and Chemical/Biological Weapons: History, Deterrence, and Arms Control, in: The Nonproliferation Review, 8. Jg. (2001) Nr. 3, S. 27-53.

Cordesman, Anthony H.: Weapons of Mass Destruction in the Middle East. Regional Trends, National Forces, Warfighting Capabilities, Delivery Options, and Weapon Effects, Center for Strategic and International Studies (CSIS), Washington, D.C. 2003.

Dando, Malcolm R.; Rappert, Brian: Codes of Conduct for the Life Sciences. Some Insights form UK Academia, in: Department of Peace Studies University of Bradford, Strengthening the Biological Weapons Convention, Briefing Paper (Second Series) Nr. 16, Mai 2005.

Dando, Malcolm: Missed Opportunities at the Chemical Weapons Treaty Meeting, in: Bulletin of Atomic Scientists, Nr. 12, Mai 2008.

Dando, Malcolm: Scientific and Technological Change and the Future of the CWC: The problem of Non-lethal Weapons, in: Disarmament Forum, Nr. 4, 2002, S. 33-44, S. 33-36.

Diab, Zuhair: Syria's Chemical and Biological Weapons. Assessing Capabilities and Motivations, in: The Nonproliferation Review, 5. Jg. (1997), Nr. 1, S. 104-111.

Haas, Peter M.: Introduction. Epistemic Communities and International Policy Coordination, in: International Organization, 46. Jg. (1992), Nr. 1, S.1-35.

45 Vgl. Meier, Oliver: CWC Review Conference Avoids Difficult Issues, in: Arms Control Today, Mai 2008. 
Herby, Peter: Protecting and Reinforcing Humanitarian Norms. The Way Forward, in: Peterson, Alan M.; Chevrier, Marie Isabelle; u.a. (Hrsg.): Incapacitating Biochemical Weapons. Lanham 2007, S. 285-291.

Justen, Detlev: Der Oslo-Prozess zum Verbot von Streumunition. Die Stigmatisierung von „Cluster Bombs“ hat begonnen, SWPStudie, Nr. 30, Berlin Oktober 2008.

Keck, Magaret; Sikkink, Kathryn: Activists Beyond Borders. Advocacy Networks in International Politics, Ithaca 1998.

Kelle, Alexander; Nixdorff, Kathryn; Dando, Malcolm: Controlling Biochemical Weapons. Adapting Multilateral Arms Control for the 21st Century, Hampshire/New York 2006.

Klotz, Lynn; Furmanski, Martin; Wheelis, Mark: Beware the Siren's Song. Why "Non-Lethal" Incapacitating Chemical Agents are Lethal, 2003, in: www.fas.org/bwc/papers/sirens_song.pdf, abgerufen am 19.11.2009.

Knauer, Sebastian: Chemiewaffen für die Bundeswehr, in: Der Spiegel, 31.07.2004.

Krieg, Kenneth J. (Under Secretary of Defense): Memorandum for Program Manager, Assembled Chemical Weapons Alternatives Deputy Under Secretary of Defense (Installations and Environment) Director, Defense Contract Management Agency (10. Januar 2007).

Meier, Oliver: CWC Review Conference Avoids Difficult Issues, in: Arms Control Today, Mai 2008

Meier, Oliver: U.S., Russia Step Up Chemical Weapons Destruction, in: Arms Control Today, Dezember 2008.

Meselson, Matthew; Robinson, Julian: „Non-lethal“ Weapons, the CWC and the BWC, in: The CBW Conventions Bulletin, Nr. 61, September 2003, S. 1-2.

OVCW: Opening Statement by the Director-General To the Executive Council at its fifty-sixth Session, 21.-24. April 2009.

OVCW: Albania - First Country to Destroy All of its Chemical Weapons. OPCW Press Release Nr. 73, Den Haag 12. Juli 2007.

Pearson, Alan M.: Incapacitating Biochemical Weapons. Science, Technology, and Policy for the 21st Century, in: The Nonproliferation Review, 13. Jg. (2006) Nr. 2, S. 151-188.

Price, Richard: A Genealogy of the Chemical Weapons Taboo, in: International Organization 49. Jg. (1995), Nr. 1, S. 73-103.
Robinson, Perry J.P.: Difficulties Facing the Chemical Weapons Convention, in: International Affairs, 84. Jg. (2008) Nr. 2, S. 223-239.

Schmidt, Helmut; Weizsäcker, Richard von; Bahr, Egon; Genscher, Hans-Dietrich: Für eine atomwaffenfreie Welt, Frankfurter Allgemeine, 09. Januar 2009.

Shultz, George P.; Perry, William J.; Kissinger, Henry A; Nunn, Sam: A World Free of Nuclear Weapons, The Wall Street Journal, 04. Januar 2007.

Seidler-Diekmann, Tabea: Alte Probleme in neuem Gewand? Herausforderungen an das umfassende Chemiewaffenverbot, HSFK-Report, 2008, Nr. 2.

Shoham, Dany: Chemical and Biological Weapons in Egypt, in: The Nonproliferation Review, 5. Jg. (1998) Nr. 3, S. 48-58.

Statement of General Thomas A. Schwartz: (Commander in Chief United Nations Command/Combined Forces Command \& Commander, Untied States Forces Korea) before the 107th USCongress Senate Armed Service Committee, 5. März 2002.

Sutherland, Ronald G.: Chemical and Biochemical Non-lethal Weapons. Political and Technical Aspects, in: SIPRI Policy Paper, Nr. 23 (2008), S. 10-11.

The International Institute for Strategic Studies: North Korea's Chemical and Biological Weapons (CBW) Programmes.

Thränert, Oliver: Zehn Jahre Chemiewaffen-Verbot. Erfahrungen und Perspektiven, in: Europäische Sicherheit, April 2007.

Thränert, Oliver; Tucker, Jonathan B.: Freeing the World of Chemical Weapons. The Chemical Weapons Convention at the Ten-year Mark, SWP Research Paper, Nr. 8, Juli 2000.

Trapp, Ralf: Advances in Science and Technology and the Chemical Weapons Convention, in: Arms Control Today, März 2008.

Tucker, Jonathan B.: Bridging the Gaps: Achieving the Potential of the Nonproliferation Treaties to Combat Nuclear, Biological, and Chemical Terrorism, in: Die Friedenswarte 83. Jg. (2008) Nr. 2-3, S. 81-103.

Tucker, Jonathan B.: War of nerves. Chemical Warfare from WW I to al-Qaeda. New York / Toronto 2006. 